\title{
COMPLICATIONS OF D-J STENT: TRAGEDY OF FRAGMENTATION- A CASE REPORT
}

\author{
MD. ASADUZZAMAN ${ }^{1}$, MD. HABIBUR RAHMAN ${ }^{1}$, MD. MOYNUL HAQUE CHOWDHURY $^{1}$, ZULKERNAIMUL $^{2}$ \\ ISLAM $^{1}$, PRANASHIS SAHA ${ }^{1}$
}

${ }^{1}$ Department of Urology, National Institute of Kidney Diseases \& Urology, Dhaka.

\begin{abstract}
Ureteric stents have been used since many years in urologic practice. Many complications encountered during its use.A 19 years old young man previously underwent an open nephrolithotomy in right side and URS with ICPL in left side to treat right renal stone \& left lower ureteric stone, respectively on the same setting. Bilateral double J-stent had been inserted at that time to provide postoperative drainage. The patient never returned for follow-up or removal of the ureteral stents. Three years later, the patient presented with a 2-week history of left lumbar pain and lower urinary tract symptoms.
\end{abstract}

Bangladesh J. Urol. 2014; 17(2): 97-100

\section{Introduction}

The first double-"J" (DJ) stent was first manufactured in 1978[1]. It provides a convenient means of drainage to the upper urinary tract. These days, DJstents are in common use because they provide efficient and relatively safe urinary diversion between the kidney and the urinary bladder[2]. We report this case to highlight the possible serious complications with long-term indwelling stents that frequently result in complications and pose a management and legal dilemma.

\section{Case Report}

A 19 years old young man previously underwent an open nephrolithotomy in right side and URS with ICPL in left side to treat right renal stone \& left lower ureteric stone, respectively on the same setting. Bilateral double J-stent had been inserted at that time to provide postoperative drainage. The patient never returned for follow-up or removal of the ureteral stents. Three years later, the patient presented with a 2-week history of left lumbar pain and lower urinary tract symptoms.

Correspondences: Md. Asaduzzaman, Department of Urology, National Institute of Kidney Diseases \& Urology, Dhaka. Emailasadnikdu@gmail.com
Physical examination was normal except a scar mark in right loin.

Laboratory study findings included normal serum creatinine. An abdominal radiograph showed forgotten multiple fragmented encrustated DJ stents on both renal tracts and bladder area(Fig.-1).

Routine biochemical parameters were anomaly free, urinalysis revealed urinary tract infection and which was managed by appropriate antibiotic.

On 22.12.13 large elongated stone over the fragmented DJ stent in urinary bladder was removed after cystolitholapaxy (Fig.-2) and fragments in lower ureter was tried to remove by URS and ICPL but attempt was not possible. Then bilateral ESWL was given to break the encrustation over the forgotten DJ stent.

On 24.01.14 ureteroscopicremoval of multiple fragmented encrustrated displaced forgotten DJ stent from both ureters were done (Fig.-3).

On 11.02.14 percutaneous nephrostomy\& retrieval of fragmented encrustated DJ stent frombilateralrenal pelvis (Fig.-5).Then patient developed urosepsis which was managed by Inj Meropenam. 


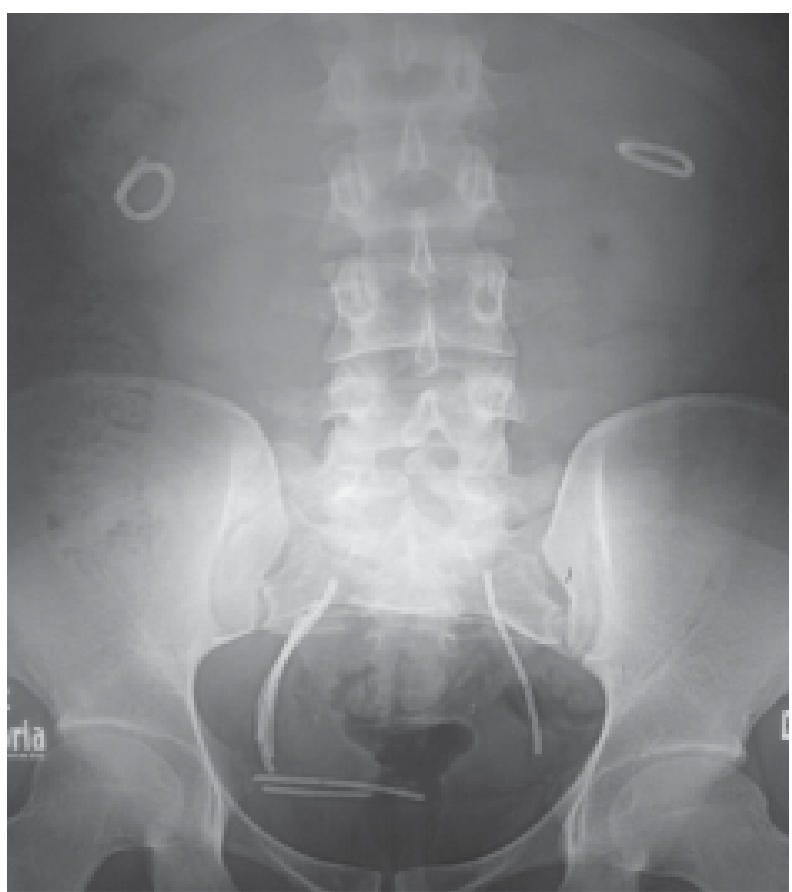

Fig.-1: Plan x-ray KUB region

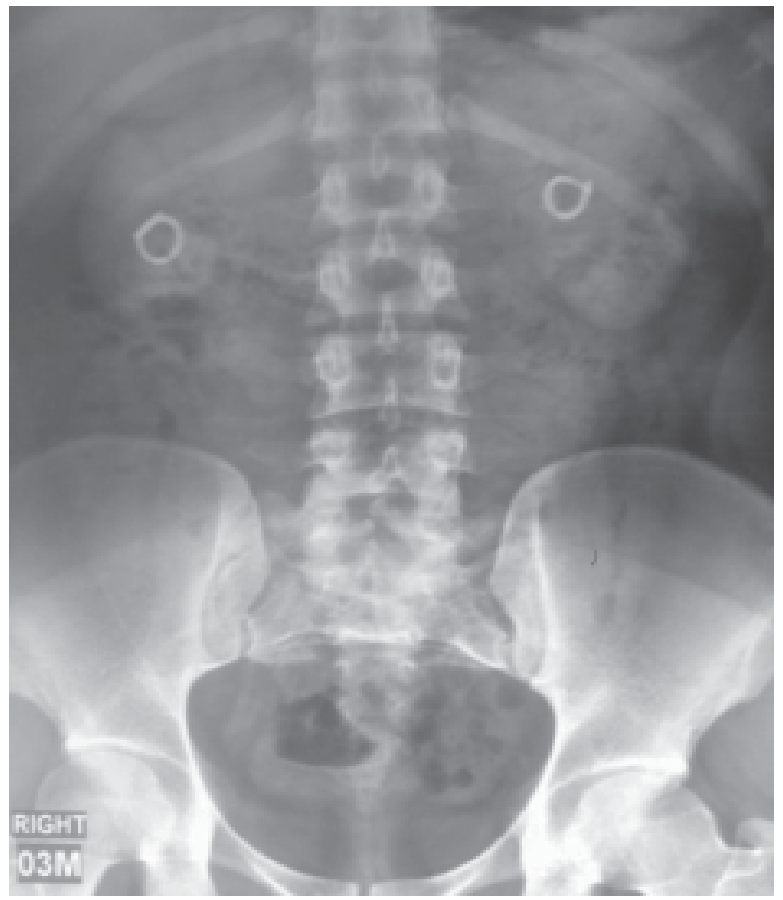

Fig.-3: After removal of fragmented DJ stent from both ureter.

\section{Discussion}

Double-J ureteral stents are widely used to provide adequate drainage of the obstructed upper urinary tract or to promote healing of ureteral lesions by preventing urinary extravasation(3). It is now a fundamental part of

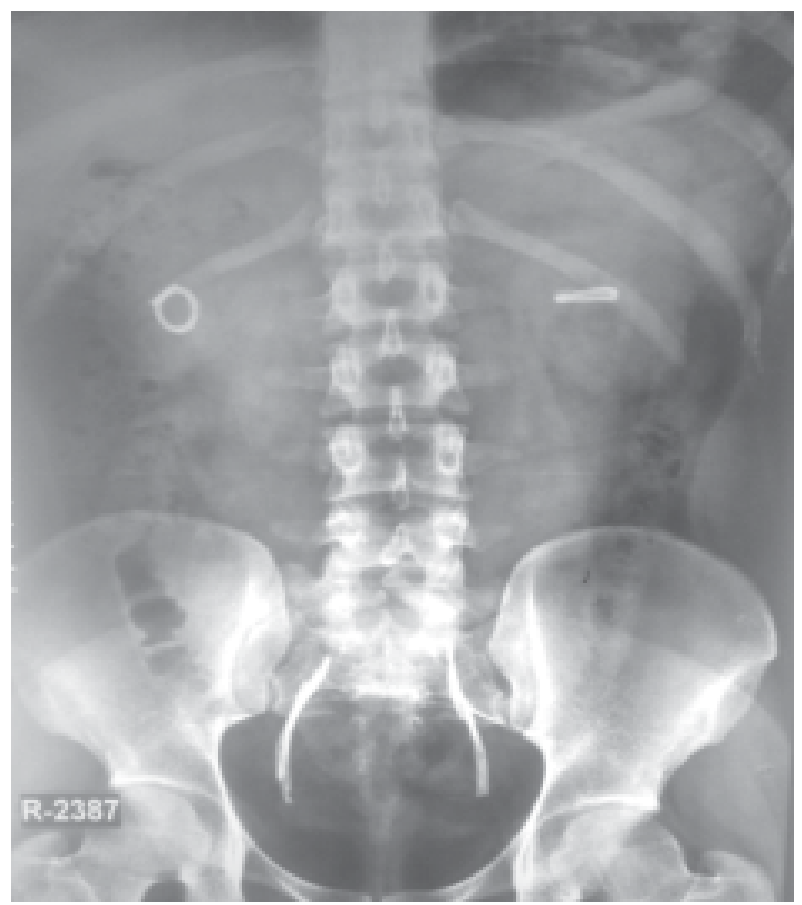

Fig.-2: Aftercystolitholapaxy

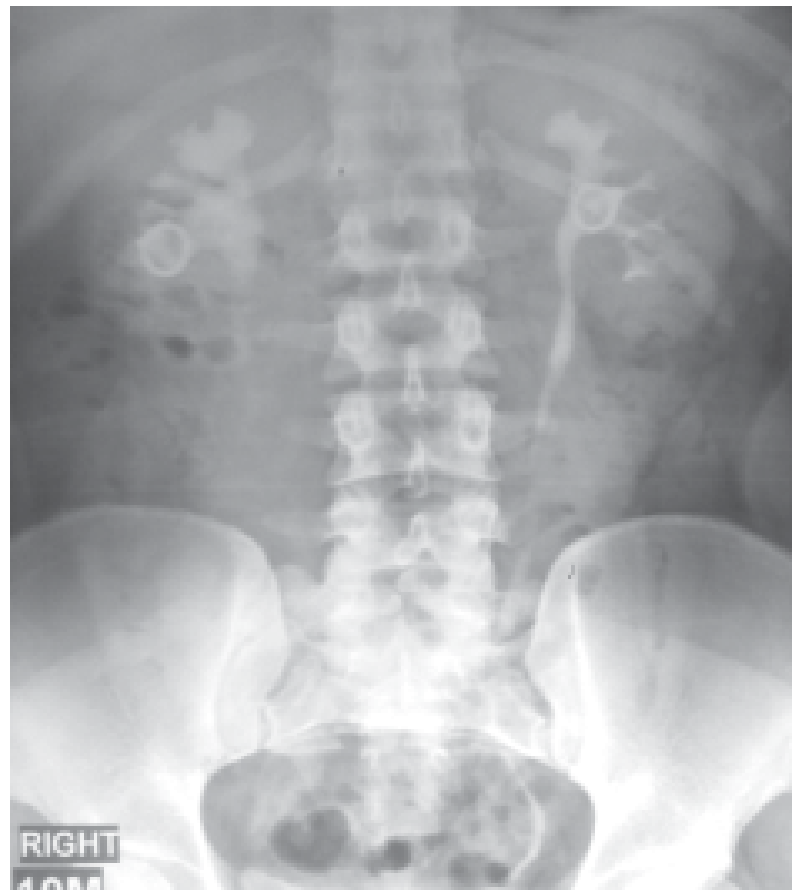

Fig.-4:IVU After removal of fragmented DJ stent from both ureter

urological practice, since its introduction in 1978, many improvements have been made in stent composition and design but complications are often encountered and could result in significant morbidity[1]. Typically ureteral stents are placed to prevent or relieve ureteral obstruction 


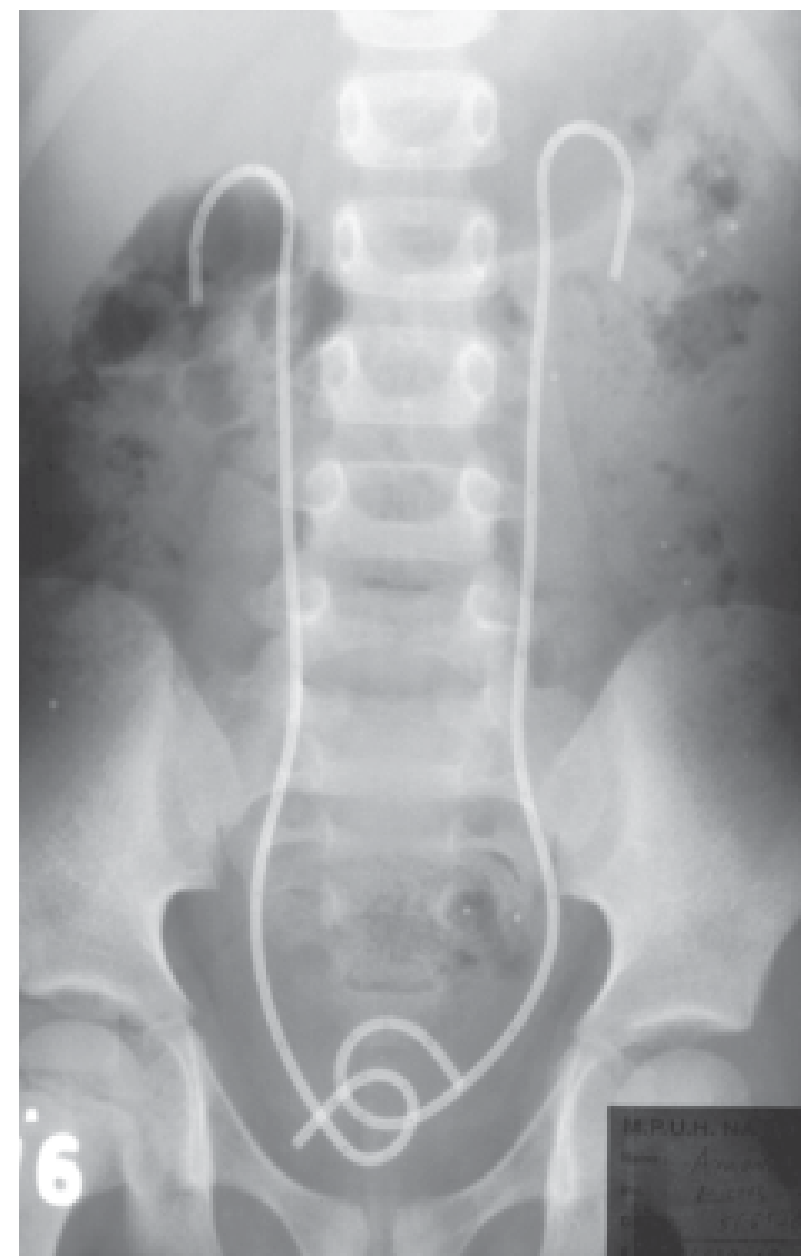

Fig.-5: After PCN and removal of $B / L$ fragmented $D J$ stent.

due to an intrinsic or extrinsic etiology, including obstructing ureteral calculi, ureteral stricture, congenital anomalies such as ureteropelvic junction obstruction, retroperitoneal tumor or fibrosis, or that developing after open or endoscopic ureteral surgery. An increasing number of double-J stents, including pure polyurethane, softened polyurethane, polyurethane-derived polymers, silicone and hydrogel-coated stents are available. Knowledge of the biocompatibility of these stents, including their effects on both the uroepithelium and the urine, should help to determine their safety, particularly for long-term use(3). With such widespread use complications have been noted, common side effects include pyelonephritis, dysuria, loin pain, hematuria, and urinary frequency and urgency. Bacteriuria is frequent, and patients often require courses of antibiotics. More serious complications are stent migration, occlusion, fragmentation, encrustation and stone formation[1]. These are well described and can lead to increased morbidity, renal impairment and repeated procedures for removal [4].Stent may become calcified, brittle and lose tensile strength and may fracture spontaneously after being in situ for a longer time[5]. Singh stated, for example, that Polyurethane stents were to prone encrustation [6]. Their higher tensile strength contributing to their rigidity may encourage encrustation; these encrustations can create a problem at the time of removal $[7,8]$. Somers developed an algorithm for evaluating and treating these complications. All patients should have intravenous urogram to determine function and to identify any obstruction. If there is no stent encrustation, a simple extraction under fluoroscopic control can be attempted. If significant stent calcification is present, extracorporeal shock-wave lithotripsy may be tried first. Open procedures are reserved for those patients with more than $3 \mathrm{~mm}$ of stent encrustation extending throughout the length of the stent, or with large volume upper tract calcification. For minimally calcified stents or for those stents with upper curls that will not straighten out on gentle traction, percutaneous extraction can be attempted in the radiology suite. If this is unsuccessful, then percutaneous nephrostolithotomy is the next step[2].

Since in our case the stent was multi-fractured with encrustation, three different approaches were carried out as described before to ensure removal of the whole stent with its casting stones. The first approach was cystolitholapaxy and removal of fragmented part of DJ stent; second onebilateral ESWL was given to broken encrustated forgotten DJ stent andremoval of multiple fragmented encrustrated displaced forgotten DJ stent from both ureters were done by URS and third one bilateral percutaneous nephrostomy \& retrieval of fragmented encrustated DJ stent from both renal pelvis.

There is still controversy about the factors predisposing encrustation. While Keane et al.(9] stated that encrustation was not associated with the level of urinary calcium. The major risk factor for stent encrustation was the presence of urolithiasis. Robert et al.[10] found that calcium oxalate was the main crystalline phase , especially in the absence of infection and suggested that prophylaxis of encrustation might consists of the preventive measures usually applied in cases of recurrent idiopathic calcium oxalate stones. According to Nakame et al.[11] encrustation and stone formation should be suspected in patients with persistent bacteriuria and/ or pyuria. The recommended replacing the ureteral stent more frequently in such patients in order to avoid complications. Sing et al. recommended the use of an 
efficient stent log under the direct supervision of the physician with patients with risk factors being monitored more frequently to avoid mishaps and morbidity[6].

\section{Conclusions}

DJ stent are an important tool in a urological armamentarium to prevent and relieve obstruction. It is very important to remember that the ureteral stent is a double edged weapon, which can behave like a friend or a foe.

Conflict of interest: None declared

\section{References}

1. Monga M, Klein E, Castaneda-Zuniga WR, Thomas $\mathrm{R}$. The forgotten indwelling ureteral stent: a urological dilemma. The Journal of urology. 1995;153(6):1817-9. .

2. Somers WJ. Management of forgotten or retained indwelling ureteral stents. Urology. 1996;47(3): 431-5.

3. Cormio L, Talja M, Koivusalo A, Makisalo H, Wolff $\mathrm{H}$, Ruutu M. Biocompatibility of various indwelling double-J stents. The Journal of urology. 1995;153(2):494-6.

4. Bultitude MF, Tiptaft RC, Glass JM, Dasgupta P. Management of encrusted ureteral stents impacted in upper tract. Urology. 2003;62(4):622-6.
5. Kehinde EO, Al-Awadi KA, Tawheed A, Al-Hunayan A, Ali Y, Mahmoud AH. Factors affecting the fate of prolonged forgotten ' $\mathrm{J}$ ' stents. Scandinavian journal of urology and nephrology. 2001;35(3): 222-7.

6. Singh I, Gupta NP, Hemal AK, Aron M, Seth A, Dogra PN. Severely encrusted polyurethane ureteral stents: management and analysis of potential risk factors. Urology. 2001;58(4):526-31.

7. Shandera KC. Minimally invasive management of the calcified ureteral stent. Techniques in urology. 1997;3(1):54-7.

8. Bukkapatnam R, Seigne J, Helal M. 1-step removal of encrusted retained ureteral stents. The Journal of urology. 2003;170(4 Pt 1):1111-4.

9. Keane PF, Bonner MC, Johnston SR, Zafar A, Gorman SP. Characterization of biofilm and encrustation on ureteric stents in vivo. British journal of urology. 1994;73(6):687-91.

10. Robert M, Boularan AM, El Sandid M, Grasset D. Double-J ureteric stent encrustations: clinical study on crystal formation on polyurethane stents. Urologia internationalis. 1997;58(2):100-4.

11. Nakame Y, Yoshida K, Takahashi T, Saitoh H, Kaneoya F, Negishi T. [Encrustation and stone formation in double $\mathrm{J}$ ureteral stent: report of two cases]. Hinyokika kiyo Acta urologica Japonica. 1986;32(6):871-5. 